\title{
Further Studies on a DTBX Prototype for the CMS Muon Detector at LHC
}

G. Barichellol, Gy.-L. Bencze ${ }^{2 \dagger}, A$. Benvenuti $^{3}, F$. Cavanna $^{4}$, M. Cuffiani ${ }^{3}$, C. Fanin ${ }^{1}$, M. De Giorgi ${ }^{1}$, P. Frabetti ${ }^{3}$, F. Gasparini ${ }^{1,2}$, R.Giantin ${ }^{1}$, I. Lippi ${ }^{1}$, S. Marcellini ${ }^{3}$, R. Martinelli ${ }^{1}$, A. Meneguzzo ${ }^{1}$, F. Navarria ${ }^{3}$, G.Piano Mortari ${ }^{4}$, G. Pitacco', E. Radermacher ${ }^{2}$, A. Rossi ${ }^{3}$, P. Sartori ${ }^{1}$, F. Szoncso ${ }^{5}$, M. Verdecchia ${ }^{4}$, C.-E. Wulz ${ }^{5}$, F. Zanchettin ${ }^{1}, P$. Zotto $^{6}$ and G. Zumerle ${ }^{1}$

1) Dip.di Fisica dell'Univ. and Sezione INFN di Padova , Italy

2) European Center for Nuclear Research (CERN), Geneva, Switzerland

3) Dip.di Fisica dell'Univ. and Sezione INFN di Bologna , Italy

4) Dip.di Fisica dell'Univ. de L'Aquila, Italy

5) Institut für Hochenergiephysik der Öst. Akad. d. Wissenschaften,Vienna, Austria

6) Dip. di Fisica del Politecnico di Milano and Sezione dell'INFN di Padova, Italy

† Visitor from Central Research Inst. for Physics, KFKI, Budapest, Hungary

\section{DFPD 94/EP/56}

(Submitted to Nuclear Instrument \& Method in Physics Research A)

UNIVERSITÀ DEGLI STLDI DI PADOVA

DIPARTIMENTO DI FISICA "GALILEO GALILEI"
ISTITUTO NAZIONALE DI FISICA NUCLEARE

SEZIONE DI PADOVA 


\title{
Further Studies on a DTBX Prototype for the CMS Muon Detector at LHC
}

\author{
G. Barichello1, Gy.-L. Bencze ${ }^{2 \dagger}$, A. Benvenuti ${ }^{3}$, F. Cavanna ${ }^{4}$, M. Cuffiani ${ }^{3}$, C. Fanin ${ }^{1}$, \\ M. De Giorgi ${ }^{1}$, P. Frabetti ${ }^{3}$, F. Gasparini ${ }^{1,2}$, R.Giantin', I. Lippi ${ }^{1}$, S. Marcellini ${ }^{3}$, \\ R. Martinelli ${ }^{1}$, A. Meneguzzol, F. Navarria ${ }^{3}$, G.Piano Mortari', G. Pitacco ${ }^{1}$,

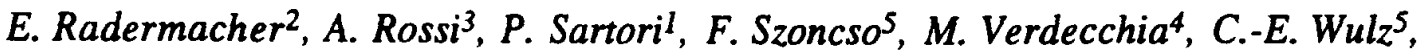 \\ F. Zanchettin ${ }^{1}, P$. Zotto $^{6}$ and G. Zumerle ${ }^{1}$
}

1) Dip.di Fisica dell'Univ. and Sezione INFN di Padova, Italy

2) European Center for Nuclear Research (CERN), Geneva, Switzeriand

3) Dip.di Fisica dell'Univ. and Sezione INFN di Bologna , Italy

4) Dip.di Fisica dell'Univ. de L'Aquila, Italy

5) Institut für Hochenergiephysik der Öst. Akad. d. Wissenschaften,Vienna, Austria

6) Dip. di Fisica del Politecnico di Milano and Sezione dell'TNFN di Padova, Italy

† Visitor from Central Research Inst. for Physics, KFKI, Budapest, Hungary

\begin{abstract}
The performance of a small prototype chamber of the baseline project for the muon barrel detector for CMS has been studied in a muon beam. Its efficiency with different gases and wire diameters, the trigger possibilities and the response in presence of a large number of electromagnetic secondaries associated to the muon are evaluated. The results are compared with a full Montecarlo simulation.
\end{abstract}




\section{1 - Introduction}

The baseline solution for the barrel muon detector of the CMS [1] experiment consists of self-triggering arrays of drift tubes called DTBX (Drift Tubes with Bunch Crossing identification). Encouraging results of a prototype exposed to a $\mathrm{SpS}$ muon beam in the RD5 experimental set-up [2] were already published [3].

However the safety requests for the LHC experimental areas make its use difficult, since the prototype was operated using $\mathrm{Ar} / \mathrm{C}_{2} \mathrm{H}_{6}$ and $\mathrm{Ar} / \mathrm{iso}-\mathrm{C}_{4} \mathrm{H}_{10}$ gas mixtures. It was then necessary to produce and test another prototype operated with non flammable gas mixtures. Meanwhile the design of the CMS muon detector became quite advanced so that it was also possible to test a configuration close to the one considered for the final detector.

\section{2 - Design Developments}

The most difficult feature required to a self-triggering device at LHC is the identification of the bunch crossing. The method studied in the previous test, already described in Ref. 2 , is based on a mean-timing operation between the drift times of three consecutive layers of staggered tubes. Using the labels of Figure 7, we define as mean-time the quantity $t_{M}=\left(t_{A}+2 t_{B}+t_{C}\right) / 2$, that is equal to the maximum drift-time of the cell, independently of the position and the angle of incidence of the track with respect to the drift tube. Therefore its computation provides the trigger signal at a fixed time interval from the muon crossing time.

The method is efficient if the single cell performance is good and, in addition, if none of the three drift times involved in the calculation is modified by the various possible background processes, otherwise the bunch crossing assignment is wrong. Unfortunately the irreducible background due to electromagnetic secondary particles production in the material ( $\delta$-ray production and muon bremsstrahlung in the absorber) is quite large for any detector, having been measured to be already about $20 \%$ at muon momentum of $100 \mathrm{Gev} / \mathrm{c}$ and slowly increasing with energy.

It is clear that with chambers composed of triplets of layers, an inefficiency or a mismeasurement in any of the three planes results in a trigger inefficiency.

In order to increase the efficiency we have therefore included a fourth layer in the DTBX design to form quadruplets of sensitive planes. In this case, the mean-timer logic requires the alignment of three out of four hits within the $25 \mathrm{~ns}$ of the LHC interbunch time, reducing the inefficiency only to situations in which data in at least two planes are missing and/or mismeasured.

The prototype test set-up is shown in Figure 1: chamber 1 was used as reference to identify background free $\mu$ tracks, while the iron, the $12 \mathrm{~cm}$ thick aluminium honeycomb and 
chambers 2 and 3 were simulating a CMS muon station.

The drift tube characteristics are described in reference 2 . The cell has a cross section of $4 \times 1 \mathrm{~cm}^{2}$, with a useful drift region of $\pm 1.9 \mathrm{~cm}$. The first chamber was equipped with 20 $\mu \mathrm{m}$ diameter W/Au wires and the others with $50 \mu \mathrm{m}$ diameter W/Au wires.

The prototype was exposed to the CERN SpS muon beam at RD5 in August 1993.

\section{3 - Prototype Performance}

\section{1 - Test with Different Gas Mixtures}

The mean-timer method can work correctly only if a strict linear relationship holds between the distance of the crossing particle from the wire and the measured drift time. This fact is guaranteed, even in a careful electrostatics design, only from the saturation of the drift velocity. Hence the choice of non-flammable gas mixtures, taking into account the large volume of gas foreseen in the final detector, is limited to $\mathrm{Ar} / \mathrm{CO}_{2}$ mixtures or to ternary mixtures with organic gases in non-explosive concentration, to enhance quenching if stable operating conditions cannot be reached.

The gas mixtures used in the test ranged from $\operatorname{Ar}(82 \%) / \mathrm{CO}_{2}(18 \%)$, to $\operatorname{Ar}(88 \%) / \mathrm{CO}_{2}(12 \%)$. The distribution of the sum of the drift-times in two consecutive staggered layers $t_{A}+t_{B}$ is shown in Figure 2a-b for a sample of normal incidence tracks, when using the two extreme gas mixtures. The degradation of the resolution employing a larger fraction of carbon-dioxide is immediately evident. This effect is explained from the fact that, for higher fractions of carbon-dioxide, the drift velocity varies along the drift path. Indeed the plot of $t_{A}$ versus $t_{B}$ for the two gases reported in Figures 3a-b shows a clear deviation from a linear correlation close to the cell walls.

The result was confirmed from calculations of the electrostatics of the drift cells performed with Finite Elements Analysis program ANSYS [4] showing that the electric field drops at its minimum close to the cathodes. As reported in literature [5], the electric field necessary to get a saturated drift velocity increases with the carbon-dioxide fraction.

\section{2 - Efficiency studies}

The efficiency of the cells was evaluated for normal incidence beam tracks counting the times a hit was found in a pre established layer when the others had fired. The efficiency curves of Figure 4 shows that a stable operating region can be reached with both wire diameters, without any organic gas addition. Figure 5 shows that the residual inefficiency is due to the cell walls, the efficiency in the sensitive region being greater than $99 \%$.

A test was also done to check the performance of the cell at high particles rates to 
search for indications of space charge effects due to the polarisation of the plastic walls. The output signal from a wire was sent to a pulse shape analyser (Lecroy 2262), sampling the signal at a frequency of $80 \mathrm{MHz}$, during a run with a high rate muon flux ( $>100 \mathrm{~Hz} / \mathrm{cm}^{2}$ ). The average maximum amplitude of the signal was measured during the beam spill (about three seconds). Figure 6 shows that no clear evidence is found for signal degradation during the spill.

\section{4 - Electromagnetic background effects}

\section{1 - Effects of $\delta$-rays Production}

The tail at low times seen in Figure 2a-b is due to hits spoiled by the electromagnetic background generated from the muon itself. We can define two categories of background events in the following way : showers (multitrack events expected to be originated in the iron block) and soft $\delta$-rays (single track events with wrong bunch crossing assignment). The amount of events falling in these categories shows a probability of about $5.5 \%$ per cell, constant with momentum, to produce a soft $\delta$ ray at a distance greater than $0.8 \mathrm{~mm}$ from the muon position ( $4 \%$ at $1.2 \mathrm{~mm}$ ) and a fraction of showers slowly rising from $5 \%$ at muon momentum of $100 \mathrm{GeV} / c$ to $8 \%$ at $300 \mathrm{GeV} / c$.

The use of a four planes DTBX makes possible a more detailed study of the effect of delta rays production. In fact, referring to Fig. 7, there is a correlation between the two quantities $t_{M 1}=\left(t_{A}+2 t_{B}+t_{C}\right) / 2$ and $t_{M 2}=\left(t_{B}+2 t_{C}+t_{D}\right) / 2$ depending on where a $\delta$-ray is produced. In fact a $\delta$-ray produced in plane $A$ or $D$ will affect only $t_{M 1}$ or $t_{M 2}$, while a $\delta$ ray produced in plane $B$ or $C$ will affect both of them by a strictly correlated amount. This is clear in Figure 8 where $t_{M 1}$ is plotted versus $t_{M 2}$ and where the events in the tail are distributed along four lines containing $\sim 80 \%$ of them. Only in the remaining $20 \%$ of the cases the two times are not correlated, indicating that the $\mu$ position measurement was spoiled in more than one plane.

\section{2 - Effect of Showers}

A deeper analysis can also be done in the showers sample to check how much of the information recorded in such events is still useful. Inside a shower events several tracks contribute to the trigger. It is not obvious that the one giving the signal at the correct time is in fact the muon. By comparison of the drift times it is possible to find how many of the hits recorded in the chambers interested from the shower belong to the actual muon track. It is found that $70 \%$ of the shower events, independently from the muon momentum, have more 
than three hits belonging to the muon track within $2.5 \sigma$ from the expected time in at least one of the two chambers downstream the iron, providing useful information to first level trigger. Considering the full sample, the probability of having at least three hits aligned in a quadruplet of planes is very high decreasing from $97.1 \%$ at $100 \mathrm{Gev} / \mathrm{c}$ to $95.3 \%$ at 300 Gev/c. The complete list is shown in Table 1: the first column refers to data collected with the iron slab removed and show that the showers are indeed originated inside the absorber.

\section{3 - Track Reconstruction}

The presence of at least three aligned hits in a quadruplet of planes is essential for triggering condition, but it can be relaxed for track fitting. Again at least three hits are required to reconstruct a muon track segment, but in this case the hits do not have to be necessarily confined in a single quadruplet of planes.

The position of the expected hits can be predicted using the clean four points tracks reconstructed in the beam defining chamber 1 . The hits are required to lie within a road of $1.3 \mathrm{~mm}$, established taking into account the chamber resolution, the uncertainties in the set-up alignment, the multiple scattering contribution and the extrapolation error. The measured probabilities of having at least $n=3, \ldots, 8$ ( out of the eight planes of a muon station) aligned hits useful for the muon track reconstruction reported in table 2.

\section{5 - Simulations}

In order to get a useful well tuned Montecarlo to be able to predict the effect of future modifications of the chamber ( e.g. chamber material or planes thickness) and to develop with a high degree of confidence the design of the tools for the CMS muon detector (e.g. the mean-timer logic chips) the prototype was carefully simulated using the GEANT[6] Montecarlo package.

The cuts applied to generate secondary particles were set to the minimum value of 10 keV kinetic energy allowed from GEANT. All known effects (beam width and dispersion, cell inefficiency, time resolution) were carefully included in the simulation. Then the above mentioned classification was applied on both simulated events and collected data samples. Table 3 shows the comparison of data with Montecarlo. It is evident that the fraction of clean muons is very well reproduced, while there is a certain trade off between the soft $\delta$-rays and the showers samples.

Figure 9 compares the distribution of $\mathrm{t}_{\mathrm{MT}}$ of the simulated events and of the collected data, showing in detail the very good agreement found on the tail of the distribution. Restricting the analysis to the soft $\delta$-rays sample it is possible to define the distance of the $\delta$ ray from the muon computing the difference between the position predicted from the three 
aligned hits and the fourth hit. The distribution is shown in Figure 10 and again the comparison with Montecario is excellent.

\section{6 - Conciusions}

The study of the new DTBX prototype confirmed the results of the first beam test, showing that good efficiency can be obtained with the use of a four layers chamber design. In addition it was shown that the detector can be safely operated with non-flammable gas mixtures. The Montecarlo simulation correctly reproduces the background seen in the data sample. The detector can be used at first level trigger with a good efficiency provided that the logic of the mean-timer electronics can recognise the alignment of three out of four possible hits in the planes of the chamber.

\section{Acknowledgements}

We wish to express our gratitude to all members of RD5 collaboration. We would like to thank V. Schiavon for the careful mounting of the electronics equipment. We also thank A. Galvani, R. Maragno and M. Zago for their work in the design and realisation of the mechanics of the prototype. We thank A. Pepato for making the ANSYS simulation of the cell. We acknowledge the help of $\mathrm{P}$. Casoli during the installation and test.

\section{References}

[1] CMS Letter of Intent, CERN LHCC 92-3, LHCC/1-1 (1992)

[2] RD5 Proposal, CERN/DRDC/90-36 (1990)

[3] F. Gasparini et al., Nucl. Instr. and Meth A 336 (1993) 91

[4] ANSYS,Finite Elements Package by Swanson Analysis System Inc.

[5] Y. Chang et al., Nucl. Instr. and Meth A 311 (1992) 490

[6] R. Brun et al., GEANT manual, CERN/DD/EE 84-1 (1987) 


\begin{tabular}{|c|r|r|r|r|}
\hline $\mathrm{p}(\mathrm{GeV} / \mathrm{c})$ & $\begin{array}{c}200 \\
\text { (No iron) }\end{array}$ & 100 & 200 & 300 \\
\hline \multicolumn{5}{|c|}{} \\
\hline three aligned hits in both quadruplets & $90.2 \%$ & $83.8 \%$ & $82.2 \%$ & $78.9 \%$ \\
\hline three aligned hits in only one quadruplet & $9.1 \%$ & $13.2 \%$ & $14.6 \%$ & $16.4 \%$ \\
\hline three aligned hits in none quadruplet & $0.6 \%$ & $2.9 \%$ & $3.2 \%$ & $4.7 \%$ \\
\hline
\end{tabular}

Table 1 - Efficiency of a mean-timer asking for three out of four aligned hits in the chamber. The first column shows results where the $20 \mathrm{~cm}$ thick iron slab was removed. The fractions are computed for tracks crossing all cells in the active region to avoid the inclusion of geometrical inefficiencies

\begin{tabular}{|c|c|c|c|}
\hline $\mathrm{p}(\mathrm{GeV} / \mathrm{c})$ & 100 & 200 & 300 \\
\hline \multicolumn{4}{|c|}{} \\
\hline $\mathrm{n}=3$ & $99.1 \%$ & $98.4 \%$ & $97.6 \%$ \\
\hline $\mathrm{n}=4$ & $96.2 \%$ & $95.2 \%$ & $94.7 \%$ \\
\hline $\mathrm{n}=5$ & $90.0 \%$ & $89.4 \%$ & $89.3 \%$ \\
\hline $\mathrm{n}=6$ & $84.1 \%$ & $82.8 \%$ & $81.6 \%$ \\
\hline $\mathrm{n}=7$ & $70.0 \%$ & $68.1 \%$ & $65.0 \%$ \\
\hline $\mathrm{n}=8$ & $45.8 \%$ & $39.3 \%$ & $34.8 \%$ \\
\hline
\end{tabular}

Table 2 - Probabilities of having at least $\mathrm{n}$ aligned hits per muon track in a chamber. The fractions are computed for tracks crossing all cells in the active region to avoid the inclusion of geometrical inefficiencies

\begin{tabular}{|c|c|c|c|c|}
\hline DATA & $\mathrm{p}(\mathrm{Gev} / \mathrm{c})$ & clean muons & soft $\delta$-rays & showers \\
\hline & $\begin{array}{l}100 \\
200 \\
300\end{array}$ & $\begin{array}{l}90.4 \% \\
88.8 \% \\
87.6 \%\end{array}$ & $\begin{array}{l}4.3 \% \\
4.5 \% \\
4.7 \%\end{array}$ & $\begin{array}{l}5.3 \% \\
6.7 \% \\
7.7 \%\end{array}$ \\
\hline \multicolumn{5}{|c|}{ MONTECARLO } \\
\hline & $\begin{array}{l}100 \\
200 \\
300\end{array}$ & $\begin{array}{l}89.9 \% \\
87.8 \% \\
85.9 \%\end{array}$ & $\begin{array}{l}3.4 \% \\
3.8 \% \\
3.9 \%\end{array}$ & $\begin{array}{r}6.7 \% \\
8.4 \% \\
10.2 \%\end{array}$ \\
\hline
\end{tabular}

Table 3 - Fraction of events per layer in the categories defined in the text for data and Montecarlo 


\section{Figure captions}

1 Experimental set-up.

2 Comparison between the sum of the drift times in two consecutive layers for two different gas mixtures.

3 Correlation of the drift times in two consecutive layers for two different gas mixtures.

4 Efficiency for two different wire diameters with as function of the high voltage applied to the anode wire. The gas mixture used was $\operatorname{Ar}(88 \%) / \mathrm{CO}_{2}(12 \%)$.

5 Efficiency as a function of the distance from the wire of the muon crossing point.

6 Average signal amplitude versus the time in the spill.

7 Mismeasurement effect of $\delta$-ray production

8 Correlation of the mean-times in the four layers DTBX.

9 Comparison of the mean-time for simulated and real data.

10 Comparison of the distance of the $\delta$-ray from the muon for simulated and real data. 


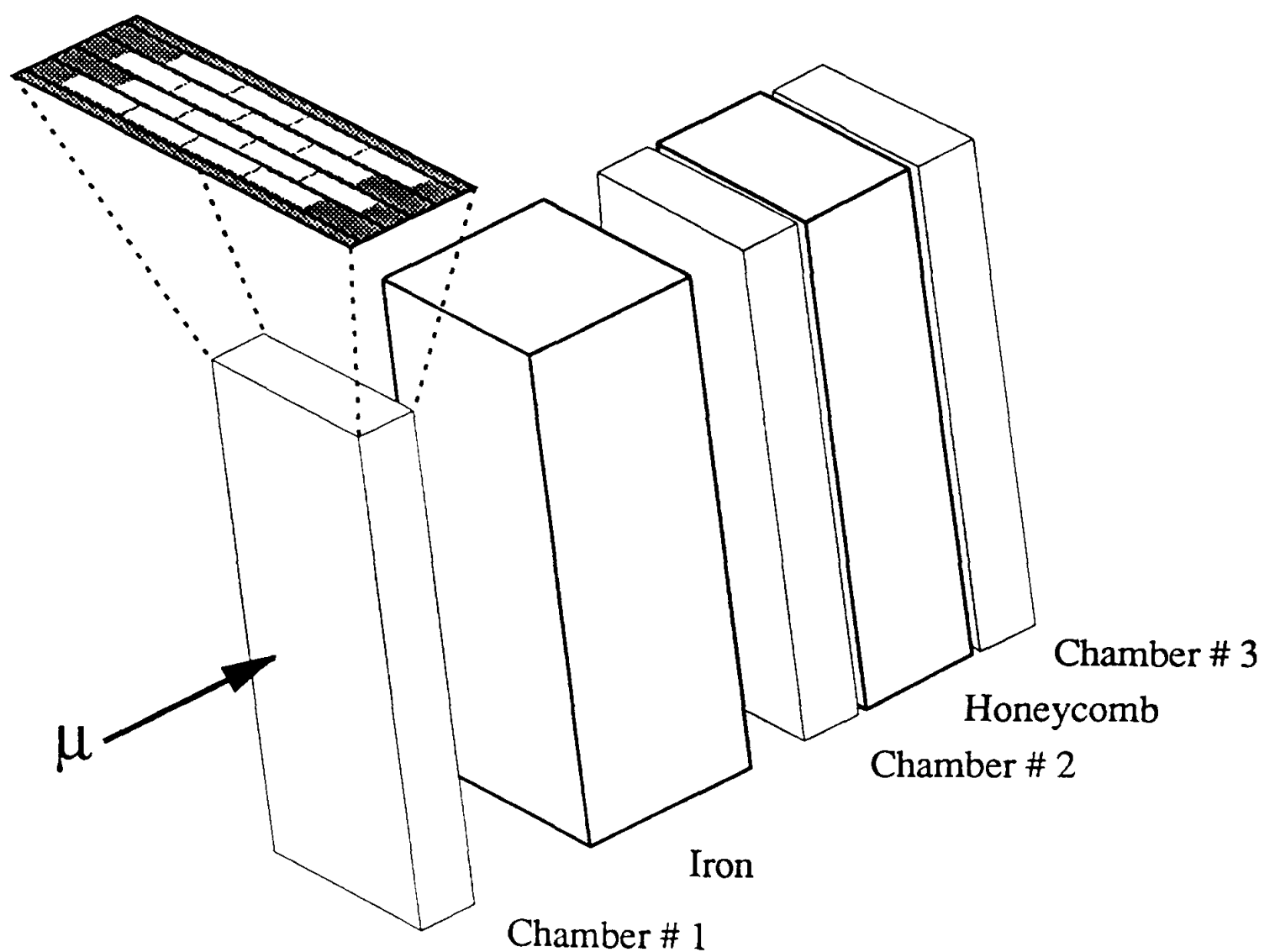

Figure 1 

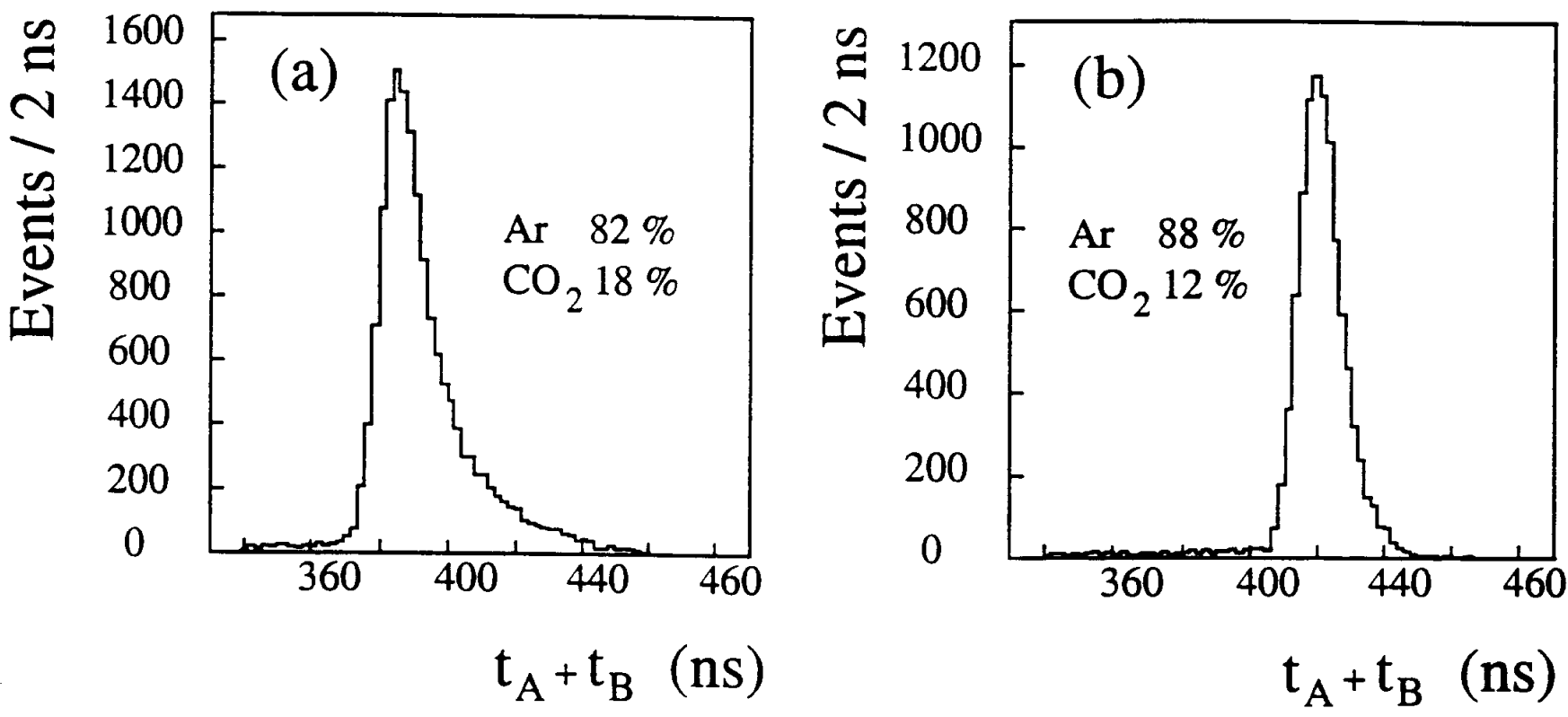

Figure 2 

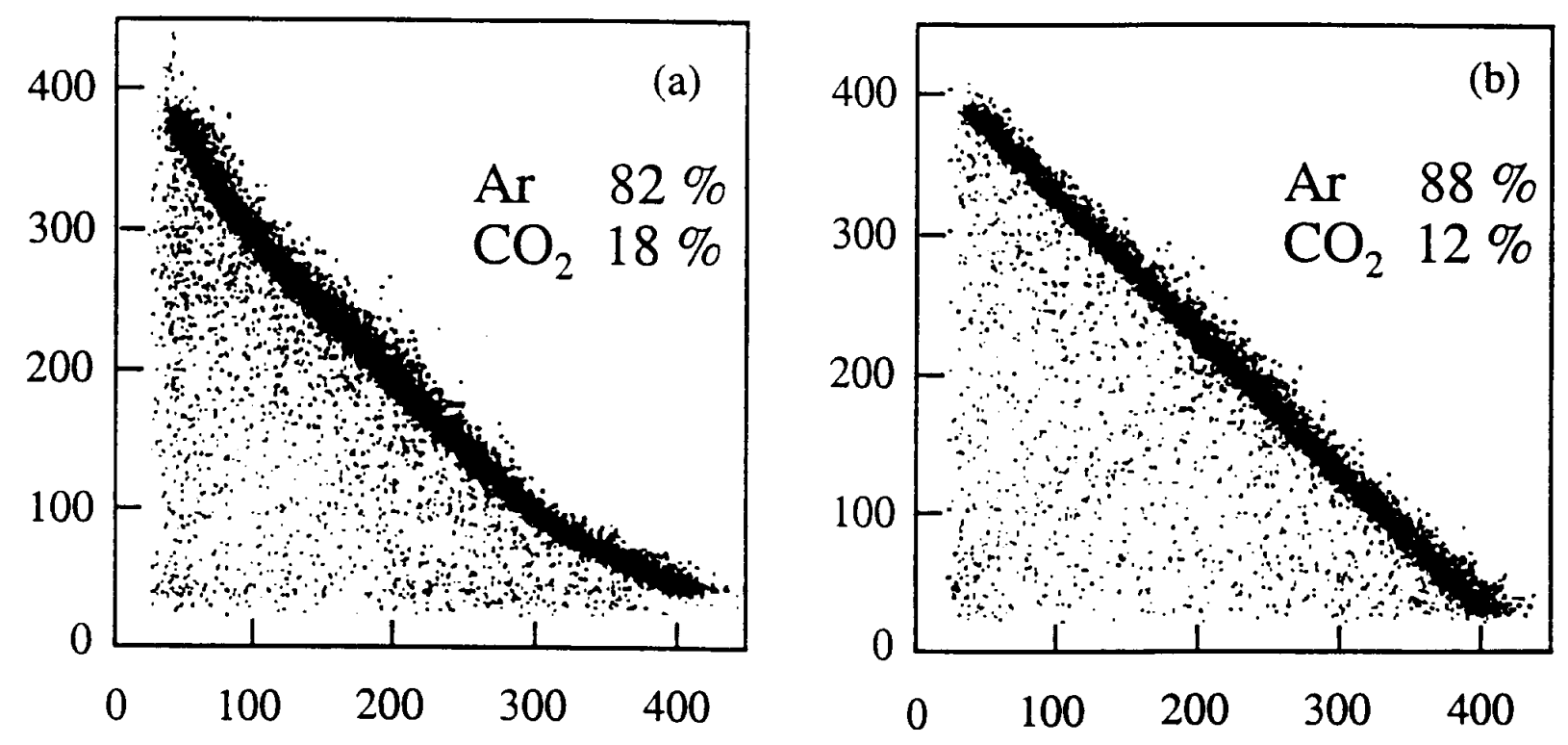

Figure 3 

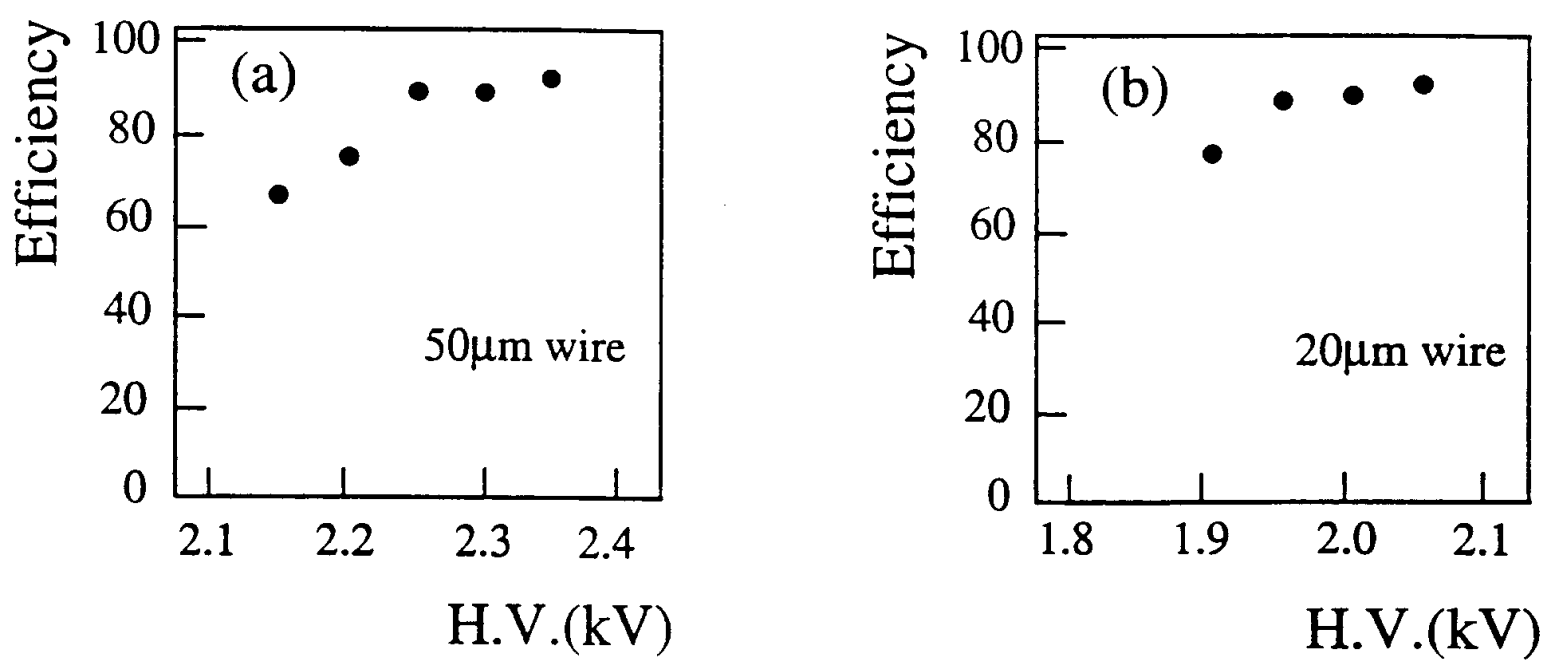

Figure 4 


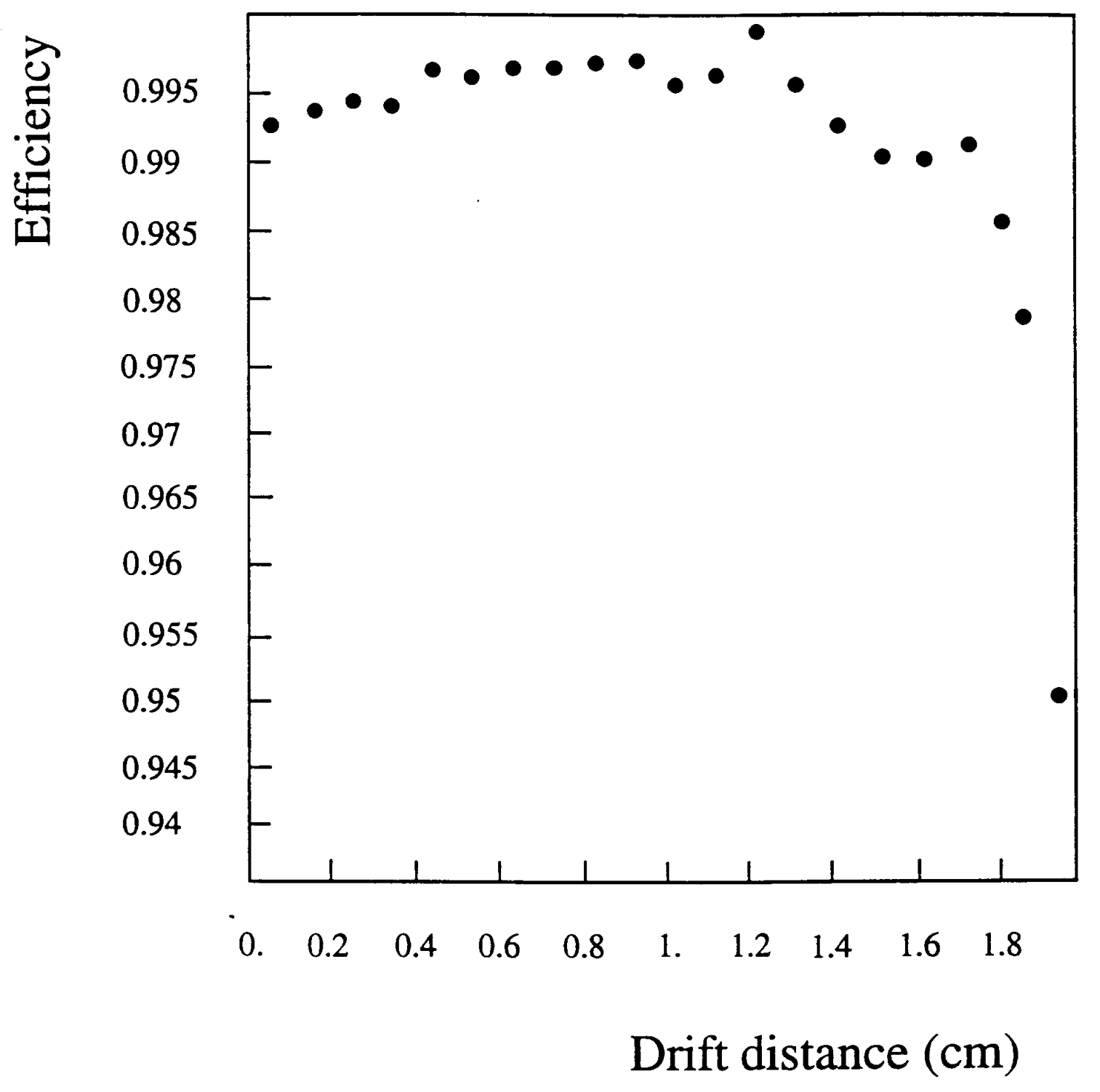

Figure 5 


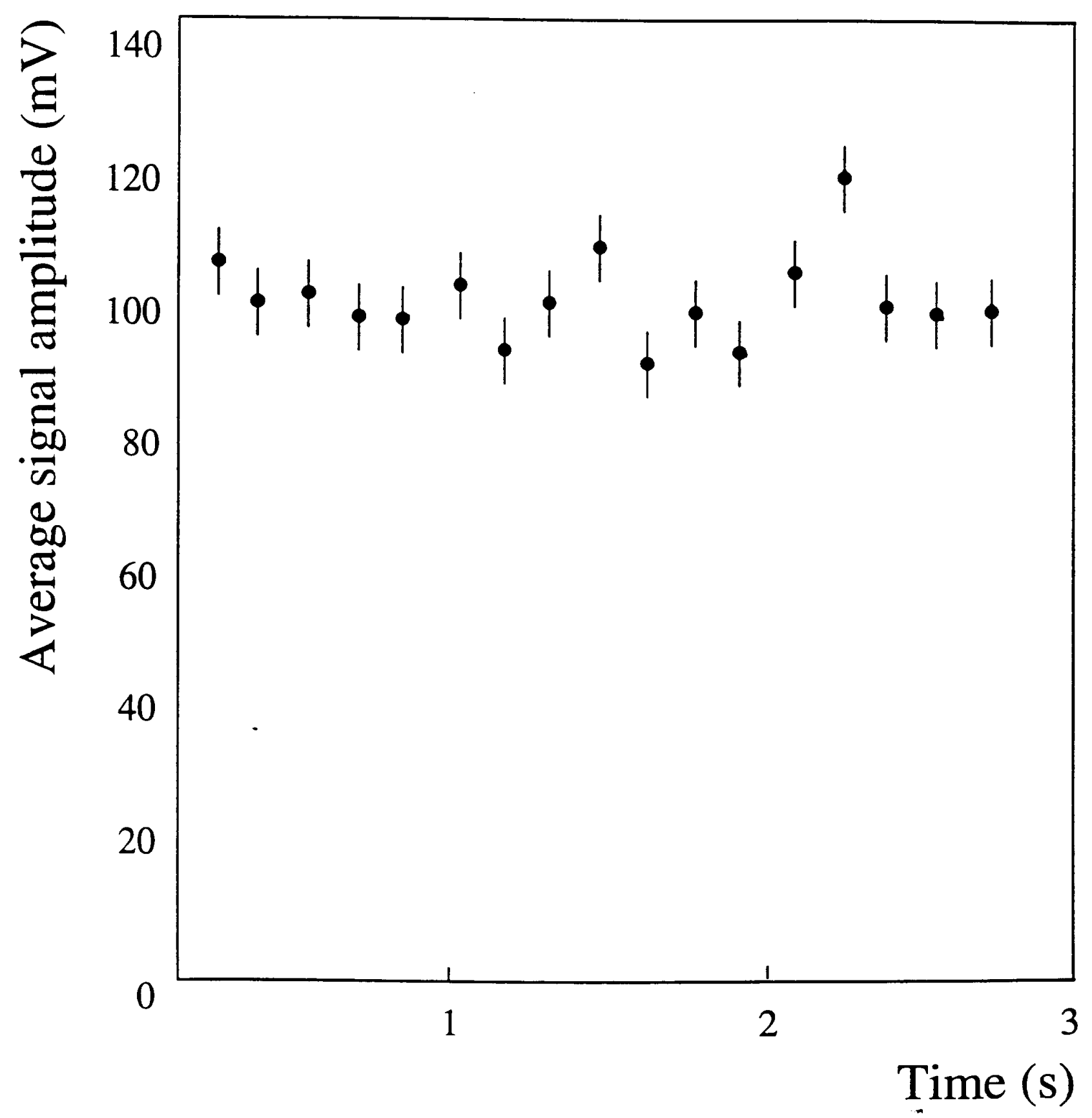

Figure 6 


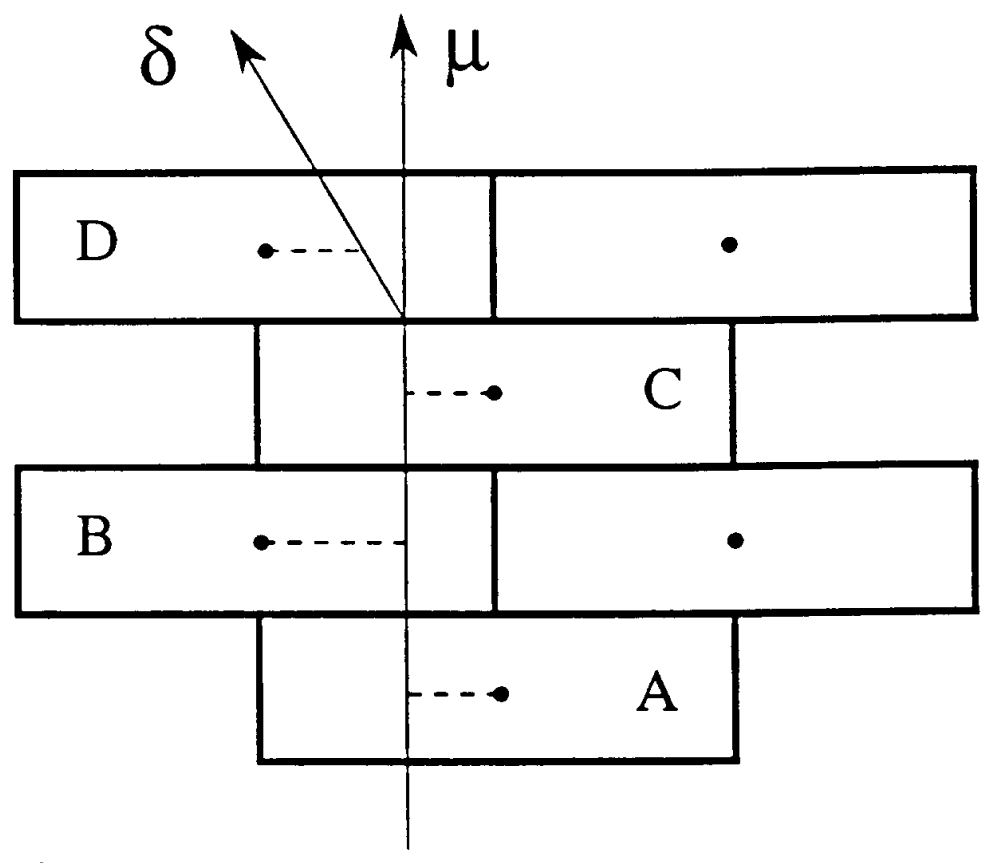

Figure 7 


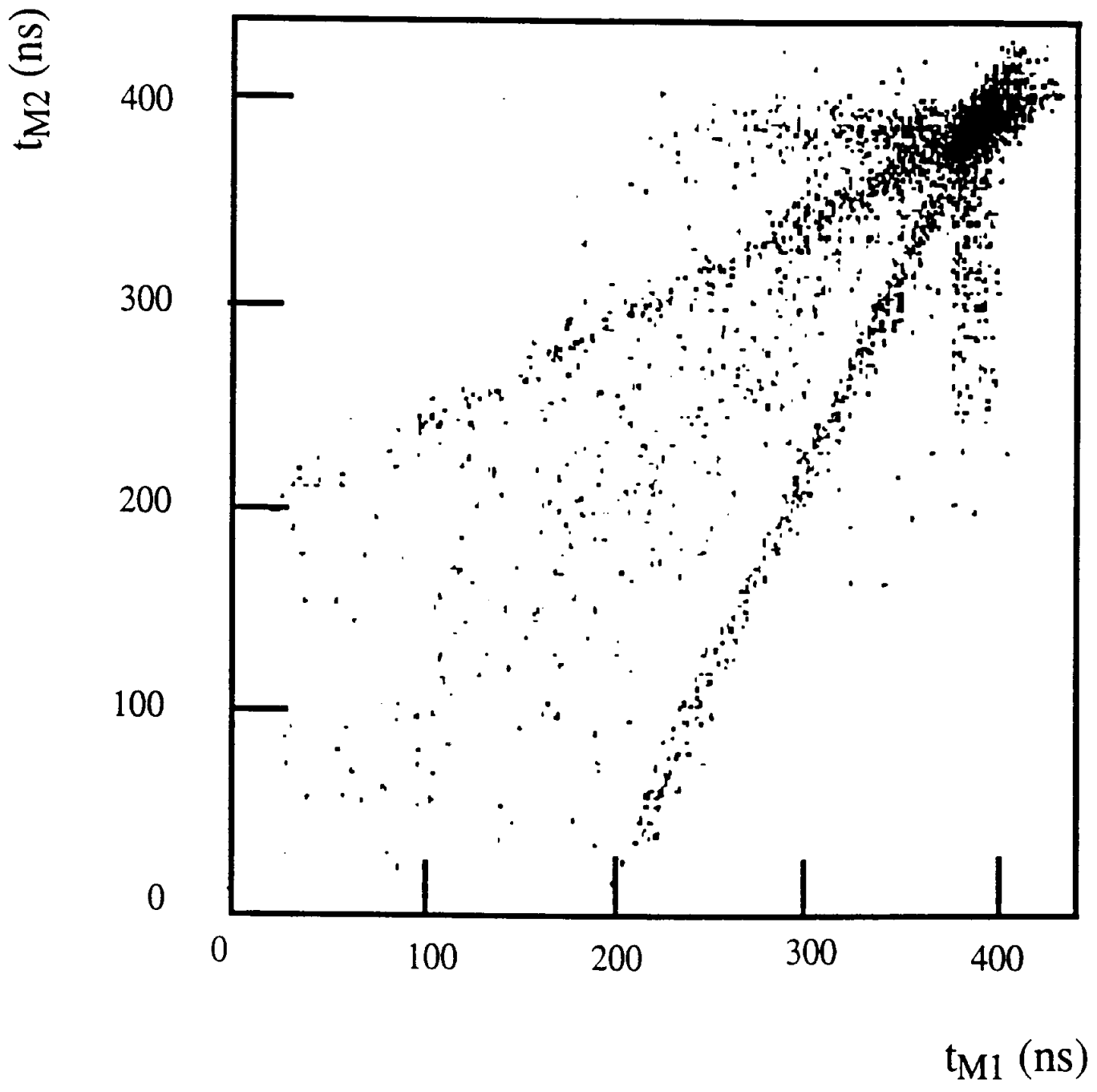

Figura 8 


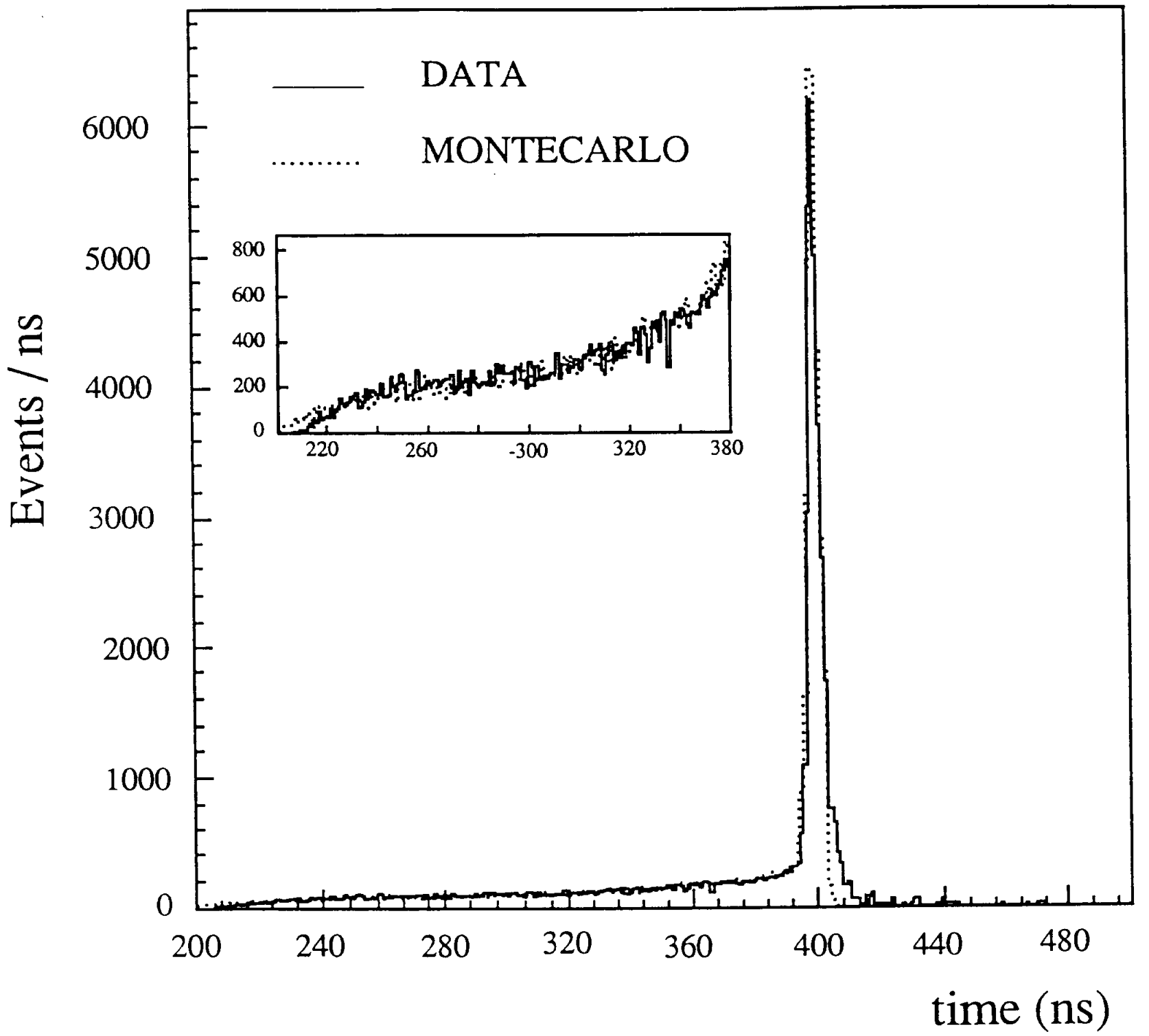

Figure 9 


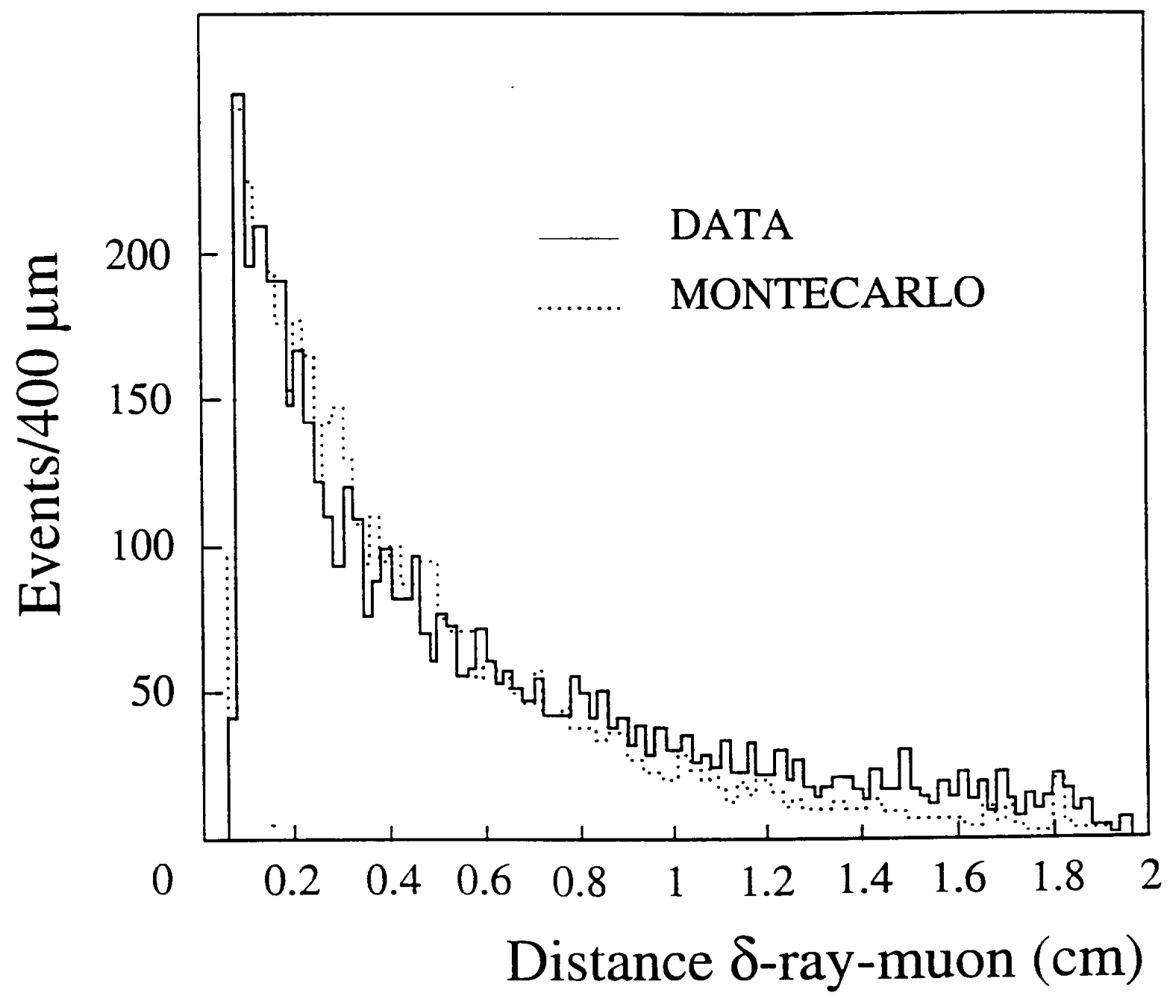

Figure 10 